\title{
BICOMPACTNESS OF CARTESIAN PRODUCTS
}

\author{
CLAUDE CHEVALLEY AND ORRIN FRINK, JR.
}

1. Introduction. Tychonoff [7] was the first to prove that the cartesian product of any number of bicompact spaces is bicompact. Of the other proofs $^{1}$ in the literature $[2,6]$ perhaps the simplest is that of Tukey, which involves the notion of an ultraphalanx. In the present note a proof of a rather general form of this theorem is given, using only simple machinery. It is shown that the same method can be used to prove that the cartesian product of any number of absolutely closed Hausdorff spaces is an absolutely closed Hausdorff space.

2. Definitions. The spaces considered are those in which an operation of closure $\bar{A}$ of a set $A$ is defined in terms of neighborhoods in the usual way, that is, $x$ is a point of $\bar{A}$ if and only if every neighborhood of $x$ contains a point of $A$. It follows that the closure operation is monotone; in other words, $\bar{A} \subset \bar{B}$ if $A \subset B$. Conversely, any closure operation which is monotone can be defined in terms of neighborhoods. No assumptions are made about the neighborhoods of a point, except that when they exist, they are sets of points.

The cartesian product $P$ of a collection of such neighborhood spaces $\left\{B_{k}\right\}$ is a space whose points $p$ are all selections $\left\{p_{k}\right\}$ containing just one point $p_{k}$ from each of the spaces $B_{k}$. Neighborhoods are defined in $P$ as follows. To any neighborhood $N_{k}$ in $B_{k}$ of a coordinate $p_{k}$ of $p$, there corresponds in the product space $P$ the neighborhood $W_{k}$ of $p$ consisting of all points $q$ of $P$ whose coordinate $q_{k}$ is in $N_{k}$. The intersection of any finite collection $\left\{W_{k_{r}}\right\}, r=1, \cdots, n$, of neighborhoods of $p$ of this type, such that no two subscripts $k_{r}$ are the same, is also defined to be a neighborhood of $p$. This is the usual definition of cartesian product due to Tychonoff [7]. Note that it is not true in general that the intersection of any finite number of neighborhoods of $p$ is a neighborhood of $p$.

A system $S$ of sets is said to have the finite intersection property if every finite number of sets of $\delta$ has at least one common point. It can be shown by a familiar argument, using Zorn's lemma or transfinite induction $[4,6,8]$, that any system $S$ of subsets of a given set

${ }^{1}$ See also J. W. Alexander, Ordered sets, complexes, and the problem of compactification, Proceedings of the National Academy of Sciences, U.S.A., vol. 25 (1939), pp. 296-298, and E. Čech, On bicompact spaces, Annals of Mathematics, (2), vol. 38 (1937), p. 830. 
with the finite intersection property is contained in a maximal system $\mathcal{X}$ with this property. This is also a consequence of the theorem of M. H. Stone that every ideal in a Boolean algebra is contained in a maximal ideal [4].

A space $B$ is said to be bicompact if there is at least one point common to the closures of the sets of any system of sets with the finite intersection property (Tukey [6]). This is equivalent in $T$-spaces to the usual definition of bicompactness.

3. Bicompactness of cartesian products. We prove the following theorem.

Theorem 1. The cartesian product $P$ of any collection of bicompact spaces $\left\{B_{k}\right\}$ is bicompact.

Proof. Let $S$ be any system of sets of $P$ with the finite intersection property, and let $\mathcal{X}$ be a maximal system with this property containing $\mathcal{S}$. Define the projection $\mathcal{X}_{k}$ of the system $\mathcal{X}$ on the space $B_{k}$ to be the system whose sets consist of the coordinates in $B_{k}$ of points of a set of $\mathcal{X}$. The system $\mathcal{X}_{k}$ clearly has the finite intersection property, since if any sets of $\mathcal{X}$ have a common point, their projections on $B_{k}$ have a common point also.

Since $B_{k}$ is bicompact, there is a point $p_{k}$ which is common to the closures of all the sets of $\mathcal{X}_{k}$. The points $\left\{p_{k}\right\}$ selected in this way, one from each space $B_{k}$, are the coordinates of a point $p$ of $P$. We wish to show that $p$ is common to the closures of all sets of $S$.

It follows from the way $p_{k}$ was selected that any neighborhood $N_{k}$ of $p_{k}$ has a point in common with each set of the system $\mathcal{X}_{k}$. Consequently in the product space $P$, the neighborhood $W_{k}$ of $p$ which corresponds to $N_{k}$, has a point in common with every set of $\mathcal{X}$. Since $\mathcal{X}$ is maximal, $W_{k}$ must belong to $\mathcal{X}$, and likewise every intersection of a finite number of such neighborhoods $\left\{W_{k_{r}}\right\}, r=1, \cdots, n$, must belong to $\mathcal{X}$. For $\mathcal{X}$, being maximal, must contain all finite intersections of its sets, since otherwise these finite intersections could be added to $\mathcal{X}$. Hence every neighborhood of $p$ belongs to $\mathcal{X}$, and therefore every neighborhood of $p$ has a point in common with every set of $\mathcal{X}$. Consequently $p$ is in the closure of every set of $\mathcal{X}$ and therefore of $\mathcal{S}$, which was to be proved.

4. Absolutely closed Hausdorff spaces. A Hausdorff space $H$ is said to be absolutely closed if every homeomorphic image of $H$ which is a subset of a Hausdorff space $K$ is a closed subset of $K[1,3,5]$.

Theorem 2. A Hausdorff space $H$ is absolutely closed if and only if 
there is a point common to the closures of the sets of any system of open sets of $H$ with the finite intersection property.

Proof. Suppose the system $S$ of open sets of $H$ has the finite intersection property, but that no point of $H$ is common to the closures of all sets of $\mathcal{S}$. Then $\mathcal{S}$ is contained in a maximal system $\mathcal{X}$ of open sets with these two properties. Extend the space $H$ to $K$ by adding to it an ideal point $x$, whose neighborhoods are all sets obtained by adding $x$ to each set of $\mathcal{X}$, while neighborhoods of points other than $x$ remain the same as in $H$. Then $K$ is a Hausdorff space, since the closures of neighborhoods of $x$ have only the point $x$ in common. Consequently $H$ is not absolutely closed, since its image in $K$ is not closed.

Conversely, suppose $H$ is not absolutely closed, but is homeomorphic to a subset $H^{*}$ of a Hausdorff space $K$, where $H^{*}$ is not closed in $K$. Let $x$ be a point of $K-H^{*}$ which is in the closure of $H^{*}$, and consider the space $H^{*} \cup\{x\}$. The system $\mathcal{X}$ of open sets of $H^{*}$ obtained by deleting $x$ from all its open neighborhoods, has the finite intersection property, since $x$ is a point of the closure of $H^{*}$. There is no point common to the closures of all the sets of $\mathscr{X}$, since $H^{*} \cup\{x\}$ is a Hausdorff space. This completes the proof.

THEOREM 3. The cartesian product $P$ of any collection of absolutely closed Hausdorff spaces $\left\{H_{k}\right\}$ is an absolutely closed Hausdorff space.

The proof is parallel to that of Theorem 1, with sets replaced by open sets. In defining the cartesian product $P$, only open neighborhoods are used, and $\mathcal{S}, \mathcal{X}$ are systems of open sets. It is merely necessary to verify that the projection on $H_{k}$ of an open set of $P$ is open.

\section{BIBLIOGRAPHY}

1. P. Alexandroff, and H. Hopf, Topologie I, Leipzig, 1935.

2. J. W. Alexander and L. Zippin, Discrete abelian groups and their character groups, Annals of Mathematics, (2), vol. 36 (1935), pp. 71-85.

3. M. Katětov, Über H-abgeschlossene und bikompakte Räume, Časopis pro Pěstováni Matematiky a Fysiky, vol. 69 (1940), pp. 36-49.

4. M. H. Stone, The theory of representations for Boolean algebras, Transactions of this Society, vol. 40 (1936), pp. 37-111.

5. - Applications of the theory of Boolean rings to general topology, Transactions of this Society, vol. 41 (1937), pp. 375-481.

6. J. W. Tukey, Convergence and Uniformity in Topology, Princeton, 1940.

7. A. Tychonoff, Über die topologische Erweiterung von Räumen, Mathematische Annalen, vol. 102 (1930), pp. 544-561.

8. H. Wallman, Lattices and topological spaces, Annals of Mathematics, (2), vol. 39 (1938), pp. 112-126.

Princeton University AND

The Institute for Advanced Study 